Pacific Journal of Mathematic 


\title{
A GENERALIZED HAUSDORFF DIMENSION FOR FUNCTIONS AND SETS
}

\author{
ROBERT J. BuCK
}

\begin{abstract}
A generalization of the Hausdorff dimension of sets is given by restricting the lengths of the intervals in the covering family. The dependence of this dimension on the choice of covering family is studied by considering the set of points in the countable unit cube $I^{\omega}$ whose coordinates are the values of the dimensions of some set for a fixed, countable collection of covering families. General conditions are given in order that two families yield the same dimension on each set, and that a covering family give the ordinary Hausdorff dimension.
\end{abstract}

In 1919, Hausdorff [3] introduced a notion of dimension for subsets of the unit interval. For any set $E$, this dimension is $H(E)=$ $\sup \left\{\gamma: \lambda_{\gamma}(E)>0\right\}$, where $\lambda_{\gamma}(E)=\inf \left\{\Sigma\left(l\left(I_{j}\right)\right)^{r}: \cup I_{j} \supseteqq E\right\}$; and it can take any value between 0 and 1 , being 1 in the case that $E$ has positive Lebesgue outer measure. This notion of dimension can be generalized in various directions and the approach taken here follows Billingsley [1]. In particular, consider the dimension $H^{\prime}(E)$ given by the outer measure $\lambda_{\gamma}^{\prime}(E)=\inf \left\{\Sigma\left(m\left(C_{i}\right)\right)^{\gamma}: \cup C_{i} \supseteqq E \& C_{i} \in \mathscr{J}\right\}$, where $m$ denotes Lebesgue measure and $\mathcal{J}$ is any collection of $m$-measurable sets containing sets of arbitrarily small measure. If $\mathcal{J}$ contains the intervals and their finite unions, then $H^{\prime}(E)$ assumes only the values 0 and 1 , as $m(E)=0$ or not. Thus for the study of sets of Lebesgue measure zero, it appears that $\mathscr{J}$ cannot be too large with respect to the family of all intervals. Accordingly, the dimension $H^{\prime}(E)$ is studied only where $\mathscr{J}$ is any collection of intervals containing intervals of arbitrarily small length and where $\mathcal{F}$ is closed under translations, i.e., where $\mathscr{J}$ is completely determined by the length of its members. Rather than use the set of these lengths to describe $\mathscr{J}$, it is more convenient to use the set $S$ of their negative logarithms, which is unbounded in $(0, \infty)$. The dimension then becomes a function $S(E)$ of the set $E$ and the unbounded set $S$. In $\S 2$, dimension is defined for a certain family $\mathscr{F}$ of nondecreasing functions, c.f. [2], [4], [5], which greatly facilitates the study.

The principal results concern the dependence of $S(E)$ on the choice of the covering set determined by $S$, and are obtained by considering the set $\mathscr{R}(S, T)$ of points in the unit square whose coordinates are respectively $S(E)$ and $T(E)$, for some set $E$. If $\Omega$ denotes the product of the closed unit interval with itself countably many times, Theorem 5 shows that the set of points in $\Omega$, whose coordinates are 
$S_{k}(E)$ for some $E$ and fixed sequence of unbounded sets $\left\{S_{k}\right\}$, is precisely the intersection of all cylinders in $\Omega$ determined by the sets $\mathscr{R}\left(S_{j}, S_{k}\right), j<k$. A characterization of $\mathscr{R}(S, T)$ directly in terms of the relative gaps in the sets $S$ and $T$ is given by Theorem 6 . The set $\mathscr{R}(S, T)$ is closed and star-shaped with respect to the diagonal $0 \leqq x=y \leqq 1$ and Theorem 7 shows that these are characteristic properties. Theorem 9 gives an especially simple necessary and sufficient condition on $S$ and $T$ for the equivalence: $S(E)=T(E)$ for all sets $E$. The remaining theorems of $\S 4$ show that for this equivalence, an unbounded set $S$ may be replaced by an increasing sequence $\left\{s_{n}\right\}$ and that $\lim s_{n+1} / s_{n}=1$ is a necessary and sufficient condition that $\left\{s_{n}\right\}$ give the ordinary Hausdorff dimension for all sets $E$.

1. Preliminaries. Let $\mathscr{F}$ be the collection of all real-valued functions $f$, defined on $(-\infty, \infty)$ with the property that $x \leqq y \rightarrow$ $0 \leqq f(y)-f(x) \leqq y-x$. The following elementary properties of $\mathscr{F}$ will be continually used without mention:

$$
\begin{aligned}
& f \in \mathscr{F} \rightarrow f+\alpha \in \mathscr{F}, \alpha \text { any constant; } \\
& f \in \mathscr{F} \text { and } 0 \leqq \alpha \leqq 1 \rightarrow \alpha f \in \mathscr{F} ; \\
& f, g \in \mathscr{F} \text { and } 0 \leqq \alpha, \beta \leqq 1, \alpha+\beta \leqq 1 \rightarrow \alpha f+\beta g \in \mathscr{F} ; \\
& \mathrm{\bigvee} f_{a} \in \mathscr{F} \text { for } f_{a} \in \mathscr{F} \text {, if } \mathrm{V} f_{a}\left(x_{0}\right)<\infty \text { for some } x_{0} ; \\
& \Lambda f_{a} \in \mathscr{F} \text { for } f_{a} \in \mathscr{F} \text {, if } \Lambda f_{a}\left(x_{0}\right)>-\infty \text { for some } x_{0} .
\end{aligned}
$$

Let $S, T$, etc., denote unbounded sets in $(0, \infty)$ and let $f \in \mathscr{F}$. Define $S(f)=\liminf f(x) / x$, over $x \rightarrow \infty, x \in S$. For $f \in \mathscr{F}, S(f)$ satisfies: $0 \leqq S(f) \leqq 1$. The number $S(f)$ is called the Hausdorff dimension of $f$ with respect to $S$. The following properties are immediate consequences of the definition:

$$
\begin{aligned}
& S\left(\Lambda f_{a}\right)=\Lambda S\left(f_{a}\right) \text { over finite collections }\left\{f_{a}\right\} \\
& S(f+\alpha)=S(f) ; \\
& S(\alpha f+\beta x)=\alpha S(f)+\beta \\
& S(f \vee \beta x)=S(f) \vee \beta .
\end{aligned}
$$

Lemma 1. Given $\varepsilon>0, f \in \mathscr{F}$, and unbounded sets $S_{1}, \cdots, S_{p}$, there is $g \in \mathscr{F}$ such that (i) $g(0) \geqq 0, g(x) \geqq\left(S_{k}(f)-\varepsilon\right) x$, for $x \in S_{k}$, $k=1,2, \cdots, p$; and (ii) $S(g)=S(f)$ for all unbounded $S$.

Proof. Choose $x_{0}>0$ large enough so that $f(x) \geqq\left(S_{k}(f)-\varepsilon\right) x$ for $x \geqq x_{0}, x \in S_{k}, k=1, \cdots, p$. Write $g(x)=(f(x) \vee 0)+x_{0}$. Then $g \in \mathscr{F}$ and $g(0) \geqq 0$. Moreover, if $0 \leqq x \leqq x_{0}$, then $g(x) \geqq x \geqq\left(S_{k}(f)-\varepsilon\right) x$. For $x \geqq x_{0}$, and $x \in S_{k}, g(x) \geqq f(x) \geqq\left(S_{k}(f)-\varepsilon\right) x$, which proves (i). 
Finally, from the construction of $g(x)$ it is clear that $S(g)=S(f)$ for all unbounded $S$.

Lemma 2. Let $f_{n} \in \mathscr{F}, n=1,2, \cdots$ and unbounded sets $S_{1}, S_{2}, \cdots$ be given. There is $f \in \mathscr{F}$ such that $S_{k}(f)=\liminf S_{k}\left(f_{n}\right)$ as $n \rightarrow \infty$, for each $k=1,2, \cdots$.

Proof. By Lemma 1, it can be assumed that for each $n, f_{n}(0) \geqq$ 0 and $f_{n}(x) \geqq\left(S_{k}\left(f_{n}\right)-\varepsilon_{n}\right) x$, for $x \in S_{k}, k \leqq n$ and $\varepsilon_{n} \rightarrow 0$ as $n \rightarrow \infty$. For each $k$ and $n$ choose $x_{n, k} \in S_{k}$ such that $x_{n, k} \rightarrow \infty$ as $n \rightarrow \infty$ and $f_{n}\left(x_{n, k}\right) \leqq\left(S_{k}\left(f_{n}\right)+\varepsilon_{n}\right) x_{n, k}$. Let $C_{n}=\bigvee_{k=1}^{n}\left(x_{n, k}-f_{n}\left(x_{n, k}\right)\right)$ and put $g_{n}(x)=$ $f_{n}(x) \vee\left(x-C_{n}\right)$. Finally write $f=\Lambda g_{n}$. Since $g_{n}(0) \geqq 0$, it follows that $f \in \mathscr{F}$. Moreover, $S_{k}\left(g_{n}\right)=1$ for each $k$ and $n$ implies $S_{k}(f)=$ $S_{k}\left(\Lambda_{n \geqq m} g_{n}\right)$ for all $m$. If $k \leqq m$, then $\Lambda g_{n} \geqq \Lambda\left(S_{k}\left(f_{n}\right)-\varepsilon_{n}\right) x$ over $n \geqq m$, so that $S_{k}(f) \geqq \lim \inf S_{k}\left(f_{n}\right)$ as $n \rightarrow \infty$. On the other hand, from the construction of $C_{n}$, it follows that for $k \leqq n, f\left(x_{n, k}\right) \leqq$ $\left(S_{k}\left(f_{n}\right)+\varepsilon_{n}\right) x_{n, k}$. Since $x_{n, k} \rightarrow \infty$ as $n \rightarrow \infty, S_{k}(f) \leqq \lim \inf S_{k}\left(f_{n}\right)$ as $n \rightarrow \infty$.

2. The Hausdorff dimension of sets. Let $\mathscr{C l}$ be the set of all continuous, real-valued, nondecreasing functions $\mu$ defined on $[0, \infty)$ such that $\mu(0)=0$ and $\mu(x)=1$, for $x \geqq 1$. Let $\mathscr{C}_{s a}$ be the subset of $\mathscr{C}$ consisting of those $\mu$ in $\mathscr{C}$ which are sub-additive, i.e., $\mu(x+y) \leqq \mu(x)+\mu(y)$. Finally, given a subset $E$ of $[0,1]$, let $\mathscr{C}(E)$ be the subset of $\mathscr{C}$ consisting of all functions $\mu$ in $\mathscr{C}$ supported by $E$, i.e., $(a, b) \cap E=\phi$ implies $\mu(a)=\mu(b)$. The set $\mathscr{L}(E)$ may be void. The operator $\Delta$, defined on $\mathscr{C}$ by $\Delta \mu(x)=\sup (\mu(y+x)-\mu(y))$ over all $y \geqq 0$, is clearly a projection of $\mathscr{l}$ onto $\mathscr{L}_{s a}$. The properties of subadditive functions needed here are given by

Lemma 3. If $\mu \in \mathscr{C}_{s a}$, then (i) $\mu(t x) \geqq \mu(x) t /(t+1)$ for $t, x \geqq 0$; and (ii) $\mu(x)>0$ for $x>0$.

Proof. If $t=0$, (i) is obvious. Otherwise

$$
\mu(x)=\mu\left(t x t^{-1}\right) \leqq\left(t x\left(1+\left[t^{-1}\right]\right)\right) \leqq\left(1+t^{-1}\right) \mu(t x),
$$

where $[z]$ denotes the greatest integer $\leqq z$. This shows (i). Part (ii) follows from (i), since $\mu(t) \geqq t /(t+1)$.

Corresponding to each $\mu$ in $\mathscr{C}$, there is $f_{\mu} \in \mathscr{F}$ defined by $f_{\mu}(x)=$ $\mathbf{V}\left(x-y-\log \Delta \mu\left(e^{-y}\right)\right)$ over $y \geqq x$. The following estimates for $f_{\mu}(x)$ will be needed:

Lemma 4. For $\mu \in \mathscr{l},-\log \Delta \mu\left(e^{-x}\right) \leqq f_{\mu}(x) \leqq \log 2-\log \Delta \mu\left(e^{-x}\right)$. 
Proof. The first inequality is trivial. By Lemma $3 \Delta \mu\left(e^{-x}\right) \leqq$ $2 e^{y-x} \Delta \mu\left(e^{-y}\right)$, which establishes the second inequality.

Using the correspondence $\mu \rightarrow f_{\mu}$, the Hausdorff dimension of functions $\mu \in \mathscr{C}$ can be defined by writing $S(\mu)=S\left(f_{\mu}\right)$, for each unbounded set $S$. Given any set $E \subseteq[0,1]$, the Hausdorff dimension of $E$ with respect to $S$ is defined to be the number:

$$
S(E)=\sup \{S(\mu): \mu \in \mathscr{C l}(E)\},
$$

taking $S(E)=0$ in the case that $\mathscr{C}(E)=\varnothing$. The connection between $S(E)$ and the classical Hausdorff dimension of $E$ is given by

THEOREM 1. ([2], [4]) $S(E)=\sup \left\{\gamma: \lambda_{S, r}(E)>0\right\}$, where $\lambda_{S, \gamma}(E)=$ $\inf \left\{\Sigma\left(l\left(I_{j}\right)\right)^{r}: \cup I_{j} \supseteqq E \&-\log l\left(I_{j}\right) \in S\right\}$.

Proof. Let $\beta<S(E)$ and $\left\{I_{k}\right\}$ be a covering of $E$ by intervals such that $-\log l\left(I_{j}\right) \in S$. By Lemmas 1 and $4,2 e^{-\beta s} \geqq \Delta \mu\left(e^{-s}\right)$ for $s \in S$ and some $\mu \in \mathscr{C}(E)$, so in particular

$$
\Sigma\left(l\left(I_{k}\right)\right)^{3} \geqq 1 / 2 \Sigma \Delta \mu\left(l\left(I_{k}\right)\right) \geqq 1 / 2 .
$$

It follows that $\lambda_{S, \lambda}(E)>0$, and hence $S(E) \leqq \sup \left\{\gamma: \lambda_{S, \gamma}(E)>0\right\}$. To show the reverse inequality, $\lambda_{S, r}(E)>0$ implies that

$$
\mu(x)=\left(\lambda_{S, \gamma}(E)\right)^{-1} \lambda_{S, r}(E \cap[0, x])
$$

belongs to $\mathscr{C}(E)$. Moreover $\mu\left(x+e^{-s}\right)-\mu(x) \leqq\left(\lambda_{S, \gamma}(E)\right)^{-1} e^{-\gamma s}$ for all $x$, so that by Lemma $3, f_{\mu}(s) / s-\left(\log \left(\lambda_{S, \gamma}(E)\right) / s \geqq \gamma\right.$ for all $s \in S$; and it follows that $S(E) \geqq \sup \left\{\gamma: \lambda_{S, \gamma}(E)>0\right\}$.

The fact that $\lambda_{s, r}$ is a sub-additive and monotone set function implies

THEOREM 2. Given any countable collection $\left\{E_{n}\right\}$ of subsets of $[0,1], S\left(\cup E_{n}\right)=\bigvee S\left(E_{n}\right)$ for all unbounded sets $S$.

Let $\mathscr{C}$ be the collection of all sets $E$ of the form: $E=\{\xi: \xi=$ $\Sigma \varepsilon_{k} \xi_{k}, \varepsilon_{k}=0$ or 1$\}$ for some positive, nonincreasing sequence $\left\{\xi_{k}\right\}$ with $\Sigma \xi_{k} \leqq 1$. For such sets $E$, the function $\mu_{E}$, defined on $[0, \infty)$ by $\mu_{E}(x)=$ $\sup \left\{\Sigma \varepsilon_{k} 2^{-k}: x \geqq \Sigma \varepsilon_{k} \xi_{k}\right\}$, belongs to $\mathscr{C}(E)$ and is sub-additive.

THEOREM 3. If $E \in \mathscr{C}$, then $S(E)=S\left(\mu_{E}\right)$ for all unbounded sets $S$.

Proof. Let $\lambda \in \mathscr{C}(E)$ and consider $s \in S$ such that $\xi_{k+1} \leqq e^{-s} \leqq$ 
$\xi_{k}$. Since $E$ is contained in the union of the $2^{k+1}$ intervals:

$$
I\left(\varepsilon_{1}, \cdots, \varepsilon_{k+1}\right)=\left[\sum_{j=1}^{k+1} \varepsilon_{j} \xi_{j}, \sum_{j=1}^{k+1} \varepsilon_{j} \xi_{j}+\xi_{k+1}\right],
$$

and any two of these intervals intersect in at most one point, it follows that $\Delta \lambda\left(e^{-s}\right) \geqq 2^{-k-1} \geqq \Delta \mu\left(e^{-s}\right) / 2$. By Lemma $4, f_{\lambda}(s) \leqq \log 4+f_{\mu}(s)$ for $s \in S$, so that $S(\lambda) \leqq S\left(\mu_{E}\right)$.

Since $S(\mu)=S\left(f_{\mu}\right)$, Theorem 3 shows that for $E \in \mathscr{C}$, there is $f \in \mathscr{F}$ such that $S(E)=S(f)$ for all $S$. The converse is also true.

THEOREM 4. For each $f \in \mathscr{F}$, there is $E_{f} \in \mathscr{C}$ such that $S(f)=$ $S\left(E_{f}\right)$ for all unbounded sets $S$.

Proof. If $f$ is bounded, then $S(f)=0$ and $E_{f}$ can be taken to be void. Thus assume $f(x) \rightarrow \infty$ as $x \rightarrow \infty$ and without loss of generality, $f(0)=0$. Select a positive, nonincreasing sequence $\xi_{k}$ satisfying $f\left(-\log \xi_{k}\right)=k \log 2$. Such sequences exist since $f$ is continuous nondecreasing and tends to $\infty$ as $x \rightarrow \infty$. Moreover, since $f(x)-x$ is nonincreasing, $\xi_{1} \leqq 1 / 2$ and $\xi_{k+1} \leqq \xi_{k} / 2$, which implies $\Sigma \xi_{k} \leqq 1$. Let $E=E_{f}$ be the set $\left\{\xi: \xi=\Sigma \varepsilon_{k} \xi_{k}, \varepsilon_{k}=0\right.$ or 1$\}$, and let $\mu=\mu_{E}$. For $s \in S$ and $\xi_{k+1} \leqq e^{-s} \leqq \xi_{k}, \log \mu\left(e^{-s}\right) \geqq-\log 2-f(s)$, so that $f(s) \geqq$ $-\log 4+f_{\mu}(s)$ by Lemma 4. Also $\log \mu\left(e^{-s}\right) \leqq \log 2-f(s)$, which shows $f(s) \leqq \log 2+f_{\mu}(s)$. Since these inequalities hold for all $s \in S$, this proves $S(f)=S(E)$.

If $\mathscr{C}_{Q^{\circ}}=\left\{\left(\alpha_{S}\right)\right.$ : for some $E \in \mathscr{C}, \alpha_{S}=S(E)$ for all $\left.S\right\}$, and if $\mathscr{R}_{S}=$ $\left\{\left(\beta_{S}\right)\right.$ : for some $f \in \mathscr{F}, \beta_{S}=S(f)$ for all $\left.S\right\}$, then Theorems 3 and 4 show $\mathscr{H}_{\mathscr{C}}=\mathscr{R}_{-}$. The situation for arbitrary subsets of $[0,1]$ is more difficult and the results are restricted to countable collections $\left\{S_{k}\right\}$ of unbounded sets.

For any pair of unbounded sets $S$ and $T$, let $\mathscr{R}(S, T)=\{(\alpha, \beta)$ : $\alpha=S(f), \beta=T(f)$ for some $f \in \mathscr{F}\}$. From the properties of $\mathscr{F}$ and $S(f)$ for $f \in \mathscr{F}$ listed in $\S 1$, it is clear that $\mathscr{R}(S, T)$ is star-shaped with respect to each point $(\alpha, \alpha), 0 \leqq \alpha \leqq 1$. Moreover, Lemma 2 implies that $\mathscr{R}(S, T)$ is always closed. Let

$$
\Omega=\left\{\left(x_{r}\right): 0 \leqq x_{r} \leqq 1, r=1,2, \cdots\right\} .
$$

For each pair of natural numbers $j, k$ with $j<k$, let $A_{j, k}$ be the cylinder in $\Omega: A_{j, k}=\left\{\left(x_{r}\right):\left(x_{j}, x_{k}\right) \in \mathscr{R}\left(S_{j}, S_{k}\right)\right\}$. Finally, let $\mathscr{H}\left[\left\{S_{k}\right\}\right]=$ $\left\{\left(\alpha_{k}\right)\right.$ : for some $\left.E \subseteq[0,1], \alpha_{k}=S_{k}(E), k=1,2, \cdots\right\}$.

THEOREM 5. Given any countable collection of unbounded sets $\left\{S_{k}\right\}, \mathscr{C}\left[\left\{S_{k}\right\}\right]=\cap A_{j, k}$ over $j<k$.

Proof. Suppose $\left(\alpha_{r}\right) \in \mathscr{H}\left[\left\{S_{k}\right\}\right]$. Let $j<k$ and $E \cong[0,1]$ such 
that $\alpha_{j}=S_{j}(E), \alpha_{k}=S_{k}(E)$. If $\alpha_{j}=\alpha_{k}$ then $\left(\alpha_{j}, \alpha_{k}\right) \in \mathscr{R}\left(S_{j}, S_{k}\right)$ so $\left(\alpha_{r}\right) \in A_{j, k}$. Thus assume $\alpha_{j} \neq \alpha_{k}$ and by symmetry, consider only the case $\alpha_{j}<\alpha_{k}$. Then given any $\varepsilon>0$, there is $f \in \mathscr{F}$ such that

$$
S_{k}(E)-\varepsilon<S_{k}(f) \leqq S_{k}(E) \text { and } S_{j}(f) \leqq S_{j}(E) \text {. }
$$

The function $g=f \vee S_{j}(E) x$ belongs to $\mathscr{F}$ and

$$
S_{j}(g)=S_{j}(E), S_{k}(E)-\varepsilon<S_{k}(g) \leqq S_{k}(E) .
$$

Since $\mathscr{R}\left(S_{j}, S_{k}\right)$ is closed, this shows $\left(\alpha_{r}\right) \in A_{j, k}$, and hence $\mathscr{H}\left[\left\{S_{k}\right\}\right] \subseteq$ $\cap A_{j, k}$ over $j<k$. Now suppose $\left(x_{r}\right) \in \cap A_{j, k}$. Then for every pair $j<k$, there is $f_{j, k} \in \mathscr{F}$ with $x_{j}=S_{j}\left(f_{j, k}\right)$ and $x_{k}=S_{k}\left(f_{j, k}\right)$. For each pair of natural numbers $p, n$, write

$$
g_{p, n}=\Lambda\left\{f_{j, k}: k=p \text { or } j=p, k+j \leqq p+n\right\} \text {. }
$$

By Lemma 2, for each $p$, there is $g_{p} \in \mathscr{F}$ such that $S_{k}\left(g_{p}\right)=\lim \inf$ $S_{k}\left(g_{p, n}\right)$ as $n \rightarrow \infty$, for each $k=1,2, \cdots$. Now write $E=\cup E_{g_{p}}$ over $p=1,2, \cdots$. By Theorems 2 and 4 , for each $k, S_{k}(E)=\bigvee S_{k}\left(g_{p}\right) \geqq$ $\lim _{n}$ inf $S_{k}\left(g_{k, n}\right)=x_{k}$. On the other hand, if $p \neq k$, then either $g_{p, n} \leqq$ $f_{k, p}$ or $g_{p, n} \leqq f_{p, k}$ for $n \geqq k$, depending whether $p<k$ or $p>k$. Thus $S_{k}(E)=S_{k}\left(g_{k}\right) \vee \bigvee_{p \neq k} \lim _{n} \inf S_{k}\left(g_{p, n}\right) \leqq x_{k}$, for each $k$, which shows $\left(x_{r}\right) \in \mathscr{H}\left[\left\{S_{k}\right\}\right]$.

In general, if the sequence $\left\{S_{k}\right\}$ contains more than two terms, the set $\mathscr{H}\left[\left\{S_{k}\right\}\right]$ properly contains the set $\left\{\left(x_{k}\right)\right.$ : for some $f \in \mathscr{F}, x_{k}=$ $\left.S_{k}(f), k=1,2, \cdots\right\}$.

3. The set $\mathscr{R}(S, T)$. The results of $\S 2$ show that the set $\mathscr{H}\left[\left\{S_{k}\right\}\right]$ is determined by the sets $\mathscr{R}\left(S_{j}, S_{k}\right), j<k$. This section lists a few of the properties of $\mathscr{R}(S, T)$. The first of these is a characterization of $\mathscr{R}(S, T)$ solely in terms of the sets $S$ and $T$.

For each $x$, let $A(x, S, T)$ consist of all pairs $(\alpha, \beta)$ with $1>\alpha \geqq$ $\beta>0$ and $(x \beta / \alpha, x(1-\beta) /(1-\alpha)) \cap S=\varnothing$. Let $B(x, S, T)$ be the set of all pairs $(\alpha, \beta)$ with $(\beta, \alpha) \in A(x, T, S)$. Finally let $\mathscr{A}(S, T)=$ $\lim \sup A(t, S, T)$ as $t \rightarrow \infty, t \in T$, and $\mathscr{B}(S, T)=\lim \sup B(s, S, T)$ as $s \rightarrow \infty, s \in S$.

THEOREM 6. For every pair of unbounded sets $S$ and $T$,

$$
\mathscr{R}(S, T)=\mathrm{C} 1(\mathscr{A}(S, T) \cup \mathscr{B}(S, T)) \text {. }
$$

Proof. Suppose $(\alpha, \beta) \in \mathscr{A}(S, T)$. If $\alpha=\beta$, then $(\alpha, \beta) \in \mathscr{R}(S, T)$. Thus assume $\beta<\alpha$. Then for some unbounded subset $T_{0}$ of $T$, the intervals $I_{t}=(t \beta / \alpha, t(1-\beta) /(1-\alpha))$ do not intersect $S$ for $t \in T_{0}$. De- 
fine a function $f$ in $\mathscr{F}$ by

$$
f(x)=\left\{\begin{array}{l}
\beta t \vee(x-(1-\beta) t), \quad \text { if } \quad x \in I_{t}, t \in T_{0} \\
\alpha x, \text { otherwise }
\end{array}\right.
$$

Then $S(f)=\alpha$ and $f(t) / t=\beta$ for $t \in T_{0}$, and so $T(f) \leqq \beta$. It follows that $\beta S(f) \geqq \alpha T(f)$ and $(1-\beta)(1-S(f)) \leqq(1-\alpha)(1-T(f))$. Since $\mathscr{R}(S, T)$ is closed and star-shaped with respect to $(0,0)$ and $(1,1)$ it follows that $\mathrm{Cl}(\mathscr{A}(S, T)) \subseteq \mathscr{R}(S, T)$. A similar argument shows $\mathrm{Cl}(\mathscr{B}(S, T)) \subseteq \mathscr{R}(S, T)$. On the other hand, let $f$ belong to $\mathscr{F}$. If $S(f)=T(f)$, then $(S(f), T(f))$, belongs to $\mathrm{Cl}(\mathscr{H}(S, T) \cup \mathscr{B}(S, T))$. Thus assume $S(f) \neq T(f)$ and by symmetry in $S$ and $T$, assume $S(f)>$ $T(f)$. It suffices to show that $S(f)>\alpha>\beta>T(f)$ implies $(\alpha, \beta) \in \mathscr{A}(S, T)$. In this case, it can be assumed by Lemma 1, that $f(s)>\alpha s$ for all $s \in S$ and that there is an unbounded subset $T_{0}$ of $T$ on which $f(t)<$ $\beta t$. Since $f \in \mathscr{F}, f(s) \leqq((s-t) \vee 0)+f(t)$ for all pairs $s$ and $t$. If $t \in T_{0}$ and $s \leqq t$, this implies $\alpha s<\beta t$. If $s \geqq t$, then $\alpha s<s-t+\beta t$. These last two inequalities imply $(t \beta / \alpha, t(1-\beta) /(1-\alpha)) \cap S=\varnothing$ or $(\alpha, \beta) \in A(t, S, T)$ for each $t \in T_{0}$. It follows that $(\alpha, \beta) \in \mathscr{A}(S, T)$.

As was noted before $\mathscr{R}(S, T)$ is always closed and star-shaped with respect to all points $(\alpha, \alpha), 0 \leqq \alpha \leqq 1$. These two properties actually characterize the shape of $\mathscr{R}(S, T)$ as is seen by

THEOREM 7. Let $\mathscr{R}$ be a closed set in the unit square, $0 \leqq$ $\alpha, \beta \leqq 1$, star-shaped with respect to $(0,0)$ and $(1,1)$. There are unbounded sets $S$ and $T$ such that $\mathscr{R}=\mathscr{R}(S, T)$.

Proof. The theorem is obvious if $\mathscr{R}$ is the diagonal $0 \leqq \alpha=\beta \leqq 1$, since for $S=T, \mathscr{R}(S, T)$ is this diagonal. Otherwise, there is a sequence $\left(\alpha_{n}, \beta_{n}\right), 0<\alpha_{n}, \beta_{n}<1, \alpha_{n} \neq \beta_{n}$ which is everywhere dense in $\mathscr{R}$. Select a sequence of intervals $\left(a_{n}, b_{n}\right)$ such that $a_{n} \rightarrow \infty$ as $n \rightarrow \infty, b_{n} \leqq a_{n+1}$ and

$$
\begin{aligned}
& b_{n} / a_{n}=\left(\alpha_{n}^{-1}-1\right) /\left(\beta_{n}^{-1}-1\right), \text { if } \alpha_{n}<\beta_{n} \\
& b_{n} / a_{n}=\left(\beta_{n}^{-1}-1\right) /\left(\alpha_{n}^{-1}-1\right), \text { if } \alpha_{n}>\beta_{n} .
\end{aligned}
$$

If $\alpha_{n}<\beta_{n}$, the interval $\left(a_{n}, b_{n}\right)$ is called an interval of type I. If $\alpha_{n}>\beta_{n}$, the interval $\left(a_{n}, b_{n}\right)$ is said to be of type II. In each interval of type I, let $s_{n}=a_{n} \beta_{n} / \alpha_{n}$, and in each interval of type II, let $t_{n}=$ $a_{n} \alpha_{n} / \beta_{n}$. In either case the constructed point belongs to $\left(a_{n}, b_{n}\right)$. Let $S$ consist of all the points $a_{n}, b_{n}$ and the points $s_{n}$. Let $T$ consist of all the points $a_{n}, b_{n}$ and the points $t_{n}$. Assume first that $(\alpha, \beta) \in \mathscr{R}$. If $\alpha=\beta$, then $(\alpha, \beta) \in \mathscr{R}(S, T)$. Thus suppose $\alpha \neq \beta$ and by symmetry in $S$ and $T$ assume $\alpha>\beta$. Select a sequence of intervals $I_{n}=$ 
$\left(a_{n}, b_{n}\right)$ of type II, such that $\alpha_{n} \rightarrow \alpha$ and $\beta_{n} \rightarrow \beta$. Define $f$ in $\mathscr{F}$ by

$$
f(x)=\left\{\begin{array}{l}
\alpha a_{n} \vee\left(x-(1-\alpha) b_{n}\right), \text { if } x \in I_{n}, n=1,2, \cdots \\
\alpha x, \text { otherwise } .
\end{array}\right.
$$

Then $S(f)=\alpha$ and for $t_{n} \in I_{n}, f\left(t_{n}\right) / t_{n}=\alpha \beta_{n} / \alpha_{n} \vee\left(1-(1-\alpha)\left(1-\beta_{n}\right) /\right.$ $\left.\left(1-\alpha_{n}\right)\right)$ which tends to $\beta$ as $n \rightarrow \infty$. Thus $T(f)=\beta$, which shows $\mathscr{R} \cong \mathscr{R}(S, T)$. To show the reverse containment it is sufficient, by Theorem 6, to show $\mathscr{A}(S, T) \subseteq \mathscr{R}$. If $(\alpha, \beta) \in \mathscr{A}(S, T)$, then for a subsequence $t_{k}$ of $\left\{t_{n}\right\},\left(t_{k} \beta / \alpha, t_{k}(1-\beta) /(1-\alpha)\right) \cap S=\varnothing$. This implies $\beta_{k} / \alpha_{k} \leqq \beta / \alpha$ and $(1-\beta) /(1-\alpha) \leqq\left(1-\beta_{k}\right) /\left(1-\alpha_{k}\right)$. Since $\mathscr{R}$ is starshaped with respect to $(0,0)$ and $(1,1)$, this shows $(\alpha, \beta) \in \mathscr{R}$.

4. Equivalence of unbounded sets. By Theorem 5 of $\S 2$ the statement, $S(E)=T(E)$ for all $E \leqq[0,1]$, is the same as, $S(f)=T(f)$ for all $f \in \mathscr{F}$. The induced equivalence relation, $S \equiv T$, deserves some study.

Theorem 8. For all unbounded sets $S, S \equiv \mathrm{Cl}(S)$.

Proof. Since $S \subseteq \mathrm{Cl}(S)$, it is clear that $S(f) \geqq \mathrm{Cl}(S)(f)$ for all $f \in \mathscr{F}$. On the other hand, there is a map $\psi: \mathrm{Cl}(S) \rightarrow S$ such that $|1-x / \psi(x)| \leqq 1 / x$ for each $x \in \mathrm{Cl}(S)$. If $f \in \mathscr{F}$, then

$$
f(s) \leqq[(s-x) \vee 0]+f(x)
$$

for every pair $s, x$. Hence $f(\psi(x)) / \psi(x) \leqq 1 / x+(1+1 / x) f(x) / x$ for all $x \in \mathrm{Cl}(S)$. It follows that $S(f) \leqq \mathrm{Cl}(S)(f)$ for $f \in \mathscr{F}$ and so $S \equiv \mathrm{Cl}(S)$.

The related partial ordering: $S \leqq T$, if and only if, $S(f) \leqq T(f)$ for all $f \in \mathscr{F}$, again equivalent to $S(E) \leqq T(E)$ for all $E \leqq[0,1]$, has the following characterization.

THEOREM 9. A necessary and sufficient condition that $S \leqq T$, is that there exist a function $\varphi: T \rightarrow S$ such that $\lim t / \varphi(t)=1$, as $t \rightarrow \infty$, $t \in T$.

Proof. If $\varphi: T \rightarrow S$ and $t / \varphi(t) \rightarrow 1$ as $t \rightarrow \infty, t \in T$, then for $f \in \mathscr{F}$, $f(\varphi(t)) \leqq[(\varphi(t)-t) \vee 0]+f(t)$, which implies

$$
f(\varphi(t)) / \varphi(t) \leqq|1-t / \varphi(t)|+(t / \varphi(t))(f(t) / t) .
$$

Hence $S(f) \leqq \varphi(T)(f) \leqq T(f)$. On the other hand, assume $S(f) \leqq T(f)$ for all $f \in \mathscr{F}$. In particular this is true for $g(x)=\mathrm{V}(s / 2 \wedge(x-s / 2))$ over $s \in \mathrm{Cl}(S)$. Here, $S(g)=1 / 2 \leqq T(g)$. For each $t \in T$, let $s(t)=$ $\sup \{s: s \in S, s \leqq t\}$ and $s^{\prime}(t)=\inf \{s: s \in S, s \geqq t\}$. Then $s(t)$ and $s^{\prime}(t)$ 
belong to $\mathrm{Cl}(S)$ and it is easy to see that $g(t)=s(t) / 2 \vee\left(t-s^{\prime}(t) / 2\right)$. Now let $\theta: T \rightarrow \mathrm{Cl}(S)$ be defined by

$$
\theta(t)=\left\{\begin{array}{l}
s(t), \text { if } t / s^{\prime}(t) \leqq s(t) / t \\
s^{\prime}(t), \text { otherwise }
\end{array}\right.
$$

If $0<\varepsilon<1 / 2$, then for $t \in T, t$ sufficiently large, $1 / 2-\varepsilon \leqq g(t) / t$, which means $1-2 \varepsilon \leqq s(t) / t$ or $s^{\prime}(t) / t \leqq 1+2 \varepsilon$. Since $\theta$ satisfies: $1 \leqq t / \theta(t) \leqq$ $s^{\prime}(t) / t$ or $1 \geqq t / \theta(t) \geqq s(t) / t$, it follows that $|1-t / \theta(t)| \leqq 2 \varepsilon$ and so $t / \theta(t) \rightarrow 1$ as $t \rightarrow \infty, t \in T$. If $\psi: \mathrm{Cl}(S) \rightarrow S$ is the mapping introduced in the proof of Theorem 8 , then the composition, $\varphi=\psi \theta$, satisfies the required property, i.e., $t / \varphi(t) \rightarrow 1$ as $t \rightarrow \infty, t \in T$.

Given any unbounded $S$, let $I_{k}=\left[n_{k}, n_{k}+1\right)$, for $n_{k}$ nonnegative integers, be a sequence of intervals such that $S \subset \cup I_{k}$ and $I_{k} \cap S$ is nonempty. Let $s_{k}=\inf \left\{s: s \in S \cap I_{k}\right\}$. Then $\left\{s_{k}\right\} \subseteq \mathrm{Cl}(S)$ and so $\left\{s_{k}\right\} \geqq$ $S$. On the other hand the map $\varphi: S \rightarrow\left\{s_{k}\right\}$ defined by $\varphi(s)=s_{k}$, if $s \in S \cap I_{k}$, clearly satisfies the condition of Theorem 9. This proves

THEOREM 10. Given any unbounded $S$, there is an increasing sequence $\left\{s_{k}\right\}$ such that $S \equiv\left\{s_{k}\right\}$.

The final result concerns the classical Hausdorff dimension $H(f)$, where $H=(0, \infty)$.

THEOREM 11. If $S=\left\{s_{n}\right\}$ and $s_{n} \leqq s_{n+1}$, then $S \equiv H$, if and only if, $\lim s_{n+1} / s_{n}=1$, as $n \rightarrow \infty$.

Proof. If $s_{n} \leqq x \leqq s_{n+1}$, then for $f \in \mathscr{F}, f\left(s_{n+1}\right) \leqq s_{n+1}-x+f(x)$, so that $f\left(s_{n+1}\right) / s_{n+1} \leqq s_{n+1} / s_{n}-1+f(x) / x$. In the case that $s_{n+1} / s_{n} \rightarrow 1$ as $n \rightarrow \infty$, it follows that $S(f) \leqq H(f)$ for all $f \in \mathscr{F}$. Since $S \leqq H$, this shows $S \equiv H$. Conversely, if $S \leqq H$, then for $g=\mathrm{V}(\alpha s \wedge(x-(1-\alpha) s)$ over $s \in S, H(g) \geqq S(f)=\alpha$, for a fixed $\alpha, 0<\alpha<1$. Thus, in particular for the points

$$
\begin{aligned}
& x_{n}=\alpha s_{n}+(1-\alpha) s_{n+1}, \lim \inf g\left(x_{n}\right) / x_{n}=\lim \inf \alpha /(\alpha+ \\
& \left.(1-\alpha) s_{n+1} / s_{n}\right) \geqq \alpha \text { as } n \rightarrow \infty \text {. Thus } s_{n+1} / s_{n} \rightarrow 1 \text { as } n \rightarrow \infty .
\end{aligned}
$$

5. Connection with other dimension functions. Dimension can be defined for more general classes of intervals, $\mathscr{J}$ cf. [1], i.e., where $\mathscr{J}$ need not be closed under translations. It is known that if $\mathcal{J}$ is the class of $r$-adic intervals, then the dimension $H^{\prime}(E)$ determined by $\mathscr{J}$ coincides with the usual Hausdorff dimension $H(E)$, 
as an easy application of Theorem 11 shows, taking

$$
S=\{-\log \mathscr{L}(I): I \in \mathscr{J}\} \text {. }
$$

For which classes $\mathscr{f}$, does the dimension $S(E)$, where

$$
S=\{-\log \mathscr{L}(I): I \in \mathscr{J}\},
$$

coincide with that determined by $\mathscr{F}$ ? More generally, for which $\mathscr{f}$, do there exist unbounded sets $S$, such that $S(E)$ coincides with $H^{\prime}(E)$ determined by $\mathscr{J}$ ? In general, the solution of these problems is not known. Notice that for such classes $\mathscr{f}$, the dimension $H^{\prime}(E)$ is necessarily a translation invariant dimension, so that one might ask if this property is also sufficient.

The author is indebted to Professor F. Bohnenblust for his advice and guidance during the preparation of this paper, which formed a part of the author's Doctoral dissertation submitted to the California Institute of Technology.

\section{BIBLIOGRAPHY}

1. P. Billingsley, Ergodic theory and information, John Wiley and Sons, Inc., New York, 1965.

2. O. Frostman, Potentiel d'équilibre et capacité des ensembles avec quelques applications à la théorie des functions, Lund. Universitet. Medd. 3 (1935), 56, 57, 85-91.

3. F. Hausdorff, Dimension und äusseres Mass, Math. Ann. 79 (1919), 157-179.

4. J. Kahane, and R. Salem, Ensembles parfaits et series trigonométriques, Hermann, Paris, 1963.

5. A. Rényi, Dimension, entropy and information, Transactions of the Second Prague Conference on Information Theory, Statistical Decision Functions, Random Processes, Academic Press, New York, 1960, 545-556.

Received January 3, 1969.

UNIVERSITY OF CALIFORNIA, DAVIS 


\section{PACIFIC JOURNAL OF MATHEMATICS}

\section{EDITORS}

H. SAMELSON

Stanford University

Stanford, California 94305

\section{RichaRd PIERCe}

University of Washington

Seattle, Washington 98105
J. DUGUNDJI

Department of Mathematics

University of Southern California

Los Angeles, California 90007

RICHARD ARENS

University of California

Los Angeles, California 90024

\section{ASSOCIATE EDITORS}

E. F. BECKENBACH

B. H. NeUManN

F. WOLF

K. YosHIDA

\section{SUPPORTING INSTITUTIONS}

UNIVERSITY OF BRITISH COLUMBIA CALIFORNIA INSTITUTE OF TECHNOLOGY UNIVERSITY OF CALIFORNIA MONTANA STATE UNIVERSITY

UNIVERSITY OF NEVADA

NEW MEXICO STATE UNIVERSITY

OREGON STATE UNIVERSITY

UNIVERSITY OF OREGON

OSAKA UNIVERSITY

UNIVERSITY OF SOUTHERN CALIFORNIA
STANFORD UNIVERSITY

UNIVERSITY OF TOKYO

UNIVERSITY OF UTAH

WASHINGTON STATE UNIVERSITY

UNIVERSITY OF WASHINGTON

${ }^{*} \quad{ }^{*} \quad{ }^{*}$
AMERICAN MATHEMATICAL SOCIETY
CHEVRON RESEARCH CORPORATION
TRW SYSTEMS
NAVAL WEAPONS CENTER




\section{Pacific Journal of Mathematics}

\section{Vol. 33, No. $1 \quad$ March, 1970}

Mir Maswood Ali, On some extremal simplexes ................... 1

Silvio Aurora, On normed rings with monotone multiplication........... 15

Silvio Aurora, Normed fields which extend normed rings of integers....... 21

John Kelly Beem, Indefinite Minkowski spaces..................... 29

T. F. Bridgland, Trajectory integrals of set valued functions ........... 43

Robert Jay Buck, A generalized Hausdorff dimension for functions and sets ......................................... 69

Vlastimil B. Dlab, A characterization of perfect rings . . . . . . . . . . . . 79

Edward Richard Fadell, Some examples in fixed point theory ............ 89

Michael Benton Freeman, Tangential Cauchy-Riemann equations and uniform approximation ............................. 101

Barry J. Gardner, Torsion classes and pure subgroups ................ 109

Vinod B. Goyal, Bounds for the solution of a certain class of nonlinear



Fu Cheng Hsiang, On C, 1 summability factors of Fourier series at a given

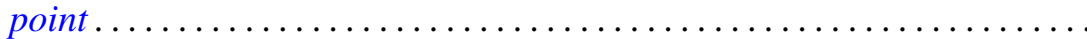

Lawrence Stanislaus Husch, Jr., Homotopy groups of PL-embedding



Daniel Ralph Lewis, Integration with respect to vector measures..........

Marion-Josephine Lim, $\mathscr{L}-2$ subspaces of Grassmann product spaces

Stephen J. Pierce, Orthogonal groups of positive definite multilinear functionals

W. J. Pugh and S. M. Shah, On the growth of entire functions of bounded index.

Siddani Bhaskara Rao and Ayyagari Ramachandra Rao, Existence of triconnected graphs with prescribed degrees . . .

Ralph Tyrrell Rockafellar, On the maximal monotonicity of subdifferential

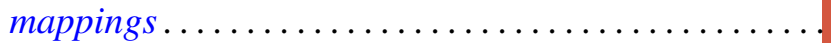

R. Shantaram, Convergence of a sequence of transformations of distribution functions. II ...............................

Julianne Souchek, Rings of analytic functions..............

Ted Joe Suffridge, The principle of subordination applied to functions of several variables...

Wei-lung Ting, On secondary characteristic classes in cobordism

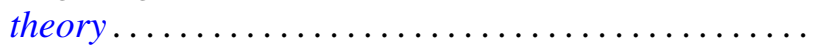

Pak-Ken Wong, Continuous complementors on $B^{*}$-algebras ...

Miyuki Yamada, On a regular semigroup in which the idempotents form a band. 